\title{
Measurement of glycosaminoglycans in canine synovial fluid and its correlation with the cause of secondary osteoarthritis, age and body weight
}

\author{
Radka Andrysíková1, Hana Kudláčková ${ }^{2}$, Miroslav Toman², Alois Nečas ${ }^{1}$ \\ ${ }^{1}$ University of Veterinary and Pharmaceutical Sciences Brno, Faculty of Veterinary Medicine, Department of \\ Surgery and Orthopaedics, Small Animal Clinic, Brno, Czech Republic \\ ${ }^{2}$ Veterinary Research Institute, Brno, Czech Republic
}

Received January 2, 2012

Accepted May 16, 2012

\begin{abstract}
Glycosaminoglycans are natural components of healthy joint cartilage and they also appear in healthy synovial fluid. An increased amount of glycosaminoglycans in synovial fluid is believed to be a marker of secondary osteoarthritis, regardless of its primary cause. The aim of our study was to define the relationship between glycosaminoglycans in the synovial fluid and joint disorders, age, and body weight. The samples of synovial fluid were obtained from dogs suffering from secondary secondary osteoarthritis $(n=35)$ and from control dogs $(n=18)$; control dogs had normal body weight. The results were compared among joints of dogs with secondary osteoarthritis divided into groups according to the criteria mentioned above and control dogs. Glycosaminoglycan concentrations in synovial fluid were measured using dimethylmethylene blue assay. The lowest mean value of glycosaminoglycans in synovial fluid was measured in the control group. Significantly higher glycosaminoglycan content $(P<0.05)$ was found in synovial fluid isolated from obese dogs compared to control dogs. Furthermore, we observed an agerelated trend, in which the highest mean values were reached either in old dogs or pups. Despite the absence of significant differences in glycosaminoglycan values among dogs suffering from various types of secondary secondary osteoarthritis, the highest mean values were measured in fragmented coronoid processus group. Our data suggest that abnormally increased body weight has an impact on glycosaminoglycan concentration in synovial fluid which may imply faster degradation and turnover of joint cartilage. Such observation has not yet been published in veterinary medicine.
\end{abstract}

Dog, joint, obesity

Glycosaminoglycans (GAG) and proteoglycans form the amorphous part of cartilage matrix, contributing to $35 \%$ of total cartilage weight. These hydrophilic molecules help counteracting mechanical pressure, thus maintaining the space structure (Kempson et al. 1970); they regulate solution penetration into cartilage (Maroudas et al. 1980) and play an important role in differentiation and regeneration of cartilage tissue (Linsenmayer and Toole 1977).

Chondroitin-6-sulphate and chondroitin-4-sulphate represent the main group of GAG and are constituent parts of aggregated proteoglycans. Another GAG representative is keratan sulphate or dermatan sulphate (Uebelhart 2008).

Regarding physiological condition, GAG or chondroitin-6-sulphate a chondroitin-4sulphate are found both in cartilage matrix and synovial fluid (SF) as they enter SF within normal cartilage turnover. Their concentration is elevated if the matrix is being degraded faster as in the case of certain pathological condition, e.g. osteoarthritis (OA). The OA development, pathophysiology, diagnostics and treatment are well reviewed by Renberg (2005). Primary OA is rarely seen in dogs; on the other hand, the secondary OA is quite common since $20 \%$ of dogs older than 1 year suffer from secondary OA (Johnston 1997). Secondary OA may result from many joint diseases such as developmental disorders (osteochondrosis - OCD, ununited anconeal process - UAP, fragmented coronoid process - FCP, hip dysplasia, patellar luxation), traumatic disorders (e. g. cranial cruciate ligament

Address for correspondence:

Radka Andrysíková

Animal Eye Center, PC

215 W 67th Ct., Loveland, CO 80503, USA
Phone: +1-9704610909;

Fax: +1-9704610027

E-mail: andrysikova@gmail.com

http://actavet.vfu.cz/ 
rupture - RCCL, intraarticular fractures) or other causes (e. g. chronic arthritis, aseptic necrosis of femoral head - Legg-Calvé-Perthes disease) (Pedersen and Poole 1978).

Changes in GAG concentration are thought to be a reliable marker of cartilage degradation level. An important factor that is necessary to objectify when comparing GAG concentration in SF is the chronicity of OA process. In early OA stages, the GAG amount may increase dramatically; however, GAG values drop slowly and approach nearly normal values in later OA stages (Innes et al. 1998). GAG can also be estimated in serum because they are filtrated through synovial membrane. Importantly, serum values may give false positive results as the half-time of GAG clearance in OA joints differs from healthy joints (Myers et al. 1996). Moreover, some authors believe GAG serum concentration is neither prognostic nor diagnostics marker of OA (Arican et al. 1994).

The physiological turnover of cartilage and its abnormal degradation during OA share some similarities. Metalloproteinases are primarily responsible for extracellular matrix degradation; also, they release proteoglycans from the complex with hyaluronic acid. Subsequently, free proteoglycans undergo proteolysis and their fragments of various size containing chondroitin sulphate, keratan sulphate, interglobular domains etc. enter SF and are further proceeded by synovial cells or removed by lymphatic vessels from the joint space. The majority of fragments enters the blood circulation and then is removed by the liver or kidneys.

The aim of our study was to prove the dependence of synovial GAG concentration on the primary cause of secondary OA as well as on the age and body weight of studied dogs.

\section{Materials and Methods}

Animals

Our study included 36 privately owned dogs treated at the Small Animal Clinic of the University of Veterinary and Pharmaceutical Sciences Brno. Thirty-one of these dogs (35 joints) suffered from joint disease and 5 (18 joints) animals were control dogs that were euthanized for disorders not affecting joints (Table 1). These control animals had healthy weight and their age ranged from 6 to 12.5 years. The diagnosis of $\mathrm{OA}$ and its underlying cause was based on history, clinical examination, radiographic assessment and perioperative findings.

Dogs with joint disease were divided into several groups based on their disease, age, and body weight. Based on disease status, dogs were divided into four groups: control dogs $(n=5)$, dogs with rupture of CCL (RCCL) $(n=18)$, dogs with FCP $(n=7)$ and dogs with other causes of OA (OCOA), such as patellar luxation, OCD or hip OA $(n=6)$. Age clustering was the following: pups (up to 6 months for small breeds, 9 months for medium breeds and 12 months for large breeds) $(n=6)$, young dogs (age 6-12 months for small breeds, 9-18 months for medium breeds and 12-30 months for large breeds) $(n=8)$, and old dogs ( 9 years or older for small breeds, 8 years or older for medium breeds and 6 years or older for large breeds) $(n=4)$. The breed category (small, medium, and large breed) is used accordingly in Table 1. Animals exceeding Fédération Cynologique Internationale (FCI) standard for given breed by $10-19 \%$ were considered overweight $(n=4)$, dogs surpassing standards by $20 \%$ or more were rated as obese $(n=5)$. Overweight and obese dogs were pooled into one group. Obese dogs were also evaluated separately.

\section{Sample collection}

Synovial fluid samples were collected from all dogs by aseptic arthrocentesis and concentrations of GAG were examined. After assessing the sample volume, heparin solution $(50 \mathrm{ml})$ was added and the sample was diluted sevenfold with phosphate-buffered saline (PBS), $\mathrm{pH}$ 7.0. The sample was then centrifuged at $200 \mathrm{~g}$ for $10 \mathrm{~min}$ at room temperature. The supernatant fluid was stored at $-80^{\circ} \mathrm{C}$ until assayed.

Dimethylmethylene blue assay for GAG concentration in SF

We used a modified colorimetric method that was based on the dimethylmethylene blue (DMMB) assay published by Arican et al. (1994). Briefly, samples of SF supernatant were sonicated (20 pulses q $0.5 \mathrm{~s}, 80 \%$ power, Branson Sonifer 150, Branson Corp., Danbury, CT, USA), transfered to a 96-well plate and incubated with $\mathrm{N}$-acetylcysteine and papain at $65^{\circ} \mathrm{C}$ for $2 \mathrm{~h}$. After digestion, iodoacetic acid, sodium chloride and DMMB were added and sample absorbance at $540 \mathrm{~nm}$ was determined by ELISA-reader iMS READER MF (LABSYSTEMS, Helsinki, Finland). Shark cartilage (BioChemika Fluka, Germany) chondroitin-6-sulphate solution was used to construct a standard curve. If not indicated otherwise, all chemicals were purchased from Sigma-Aldrich (Prague, Czech Republic). 
Statistical analyses

The normality of data distribution was tested first. The criterion of normality was not fulfilled hence the data were evaluated by the non-parametric Kruskal-Wallis test. The $P$ values lower than 0.05 were considered significant.

\section{Results}

The GAG concentrations were measured in SF samples obtained from 53 joints (36 dogs) of which 18 joints were used as control samples. The results were compared among dog groups divided according to given criteria (disease status, age, body weight) and control dogs. The GAG found in SF together with other variables characterizing the dogs are shown in Table 1.

The GAG concentrations in SF compared among groups of dogs with different disease status are shown in Fig. 1. The mean values of GAG concentrations in SF were following: $1.14 \pm 0.58 \mathrm{mg} / \mathrm{ml}, 1.43 \pm 1.088 \mathrm{mg} / \mathrm{ml}, 1.81 \pm 1.51 \mathrm{mg} / \mathrm{ml}$, and $2.87 \pm 2.43 \mathrm{mg} / \mathrm{ml}$ in the control group, RCCL, OCOA, and FCP groups, respectively.

The GAG concentrations in SF compared among age groups of dogs are presented in Fig. 2. The mean values of GAG concentrations in SF were following: $1.14 \pm 0.58 \mathrm{mg} / \mathrm{ml}$, $1.22 \pm 0.51 \mathrm{mg} / \mathrm{ml}, 1.44 \pm 1.42 \mathrm{mg} / \mathrm{ml}, 2.50 \pm 2.59 \mathrm{mg} / \mathrm{ml}$, and $2.71 \pm 1.79 \mathrm{mg} / \mathrm{ml}$ in the control group, the group of young animals, adult dogs, pups, and old dogs, respectively.

The GAG concentrations in SF compared among body weight groups are presented in Fig. 3. The mean values of GAG concentrations in SF were following: $1.14 \pm 0.58 \mathrm{mg} / \mathrm{ml}$, $1.80 \pm 0.73 \mathrm{mg} / \mathrm{ml}, 1.80 \pm 0.58 \mathrm{mg} / \mathrm{ml}$, and $2.85 \pm 1.80 \mathrm{mg} / \mathrm{ml}$ in the control group, group of dogs with healthy weight, overweight group including obese dogs, and obese group, respectively. The mean value of GAG concentration in SF measured in the obese group was significantly higher compared to control dogs $(P<0.05)$.

\section{Discussion}

Secondary OA is one of the most common orthopaedic disorders in dogs. Despite the fact that secondary $\mathrm{OA}$ is a common result of abnormal development of joint structures (elbow or hip dysplasia, FCP, UAP etc.), the extending lifespan, increasing number of

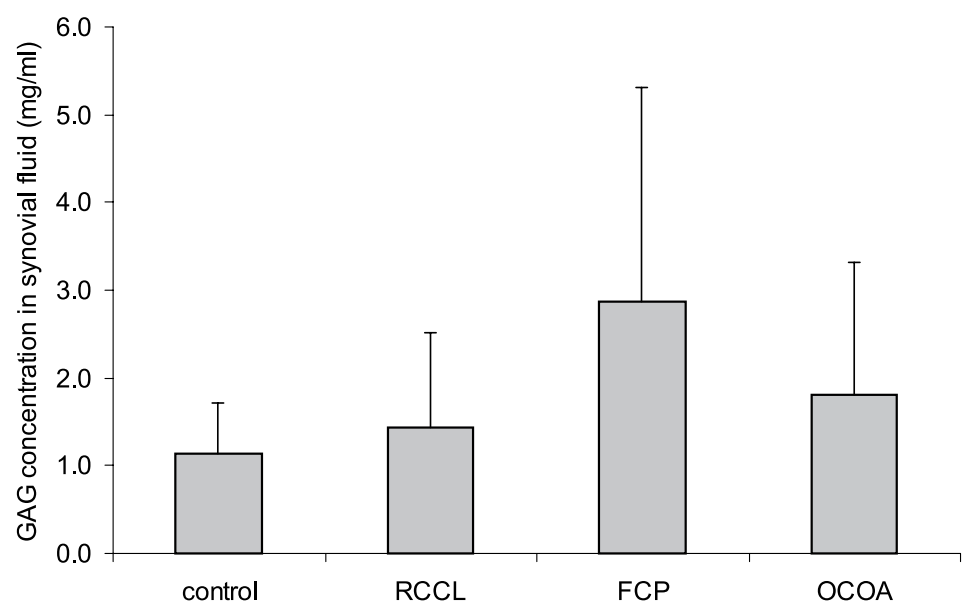

Fig. 1. Comparison of glycosaminoglycan (GAG) mean values among groups of dogs based on disease status. Control - dogs euthanized for disorders not affecting joints, $n=18$ (joints); RCCL - dogs with ruptured cranial cruciate ligament, $\mathrm{n}=18$ (joints); $\mathrm{FCP}$ - dogs with fragmented coronoid process, $\mathrm{n}=10$ (joints); OCOA - dogs with other causes of osteoarthritis, $\mathrm{n}=7$ (joints). 


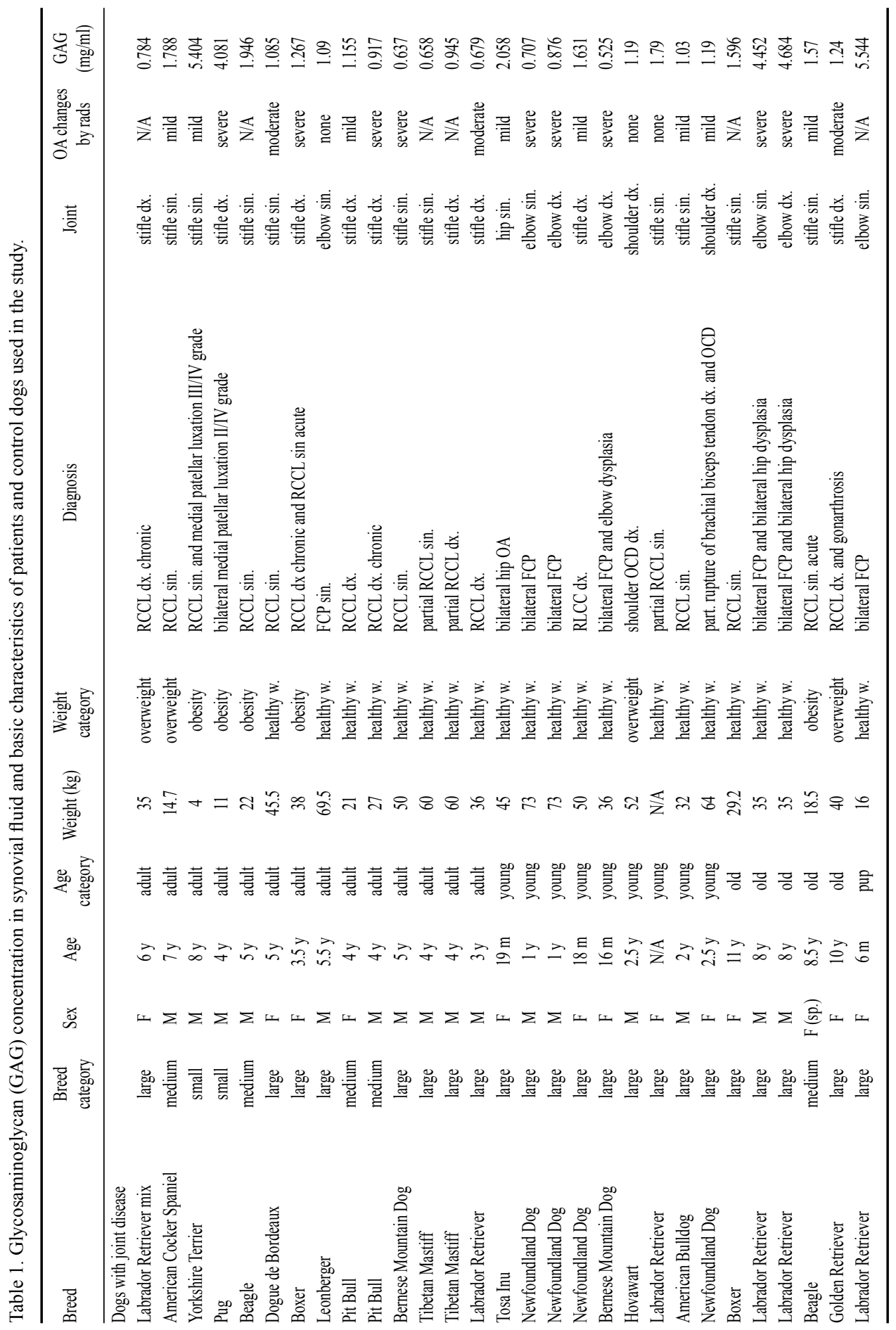




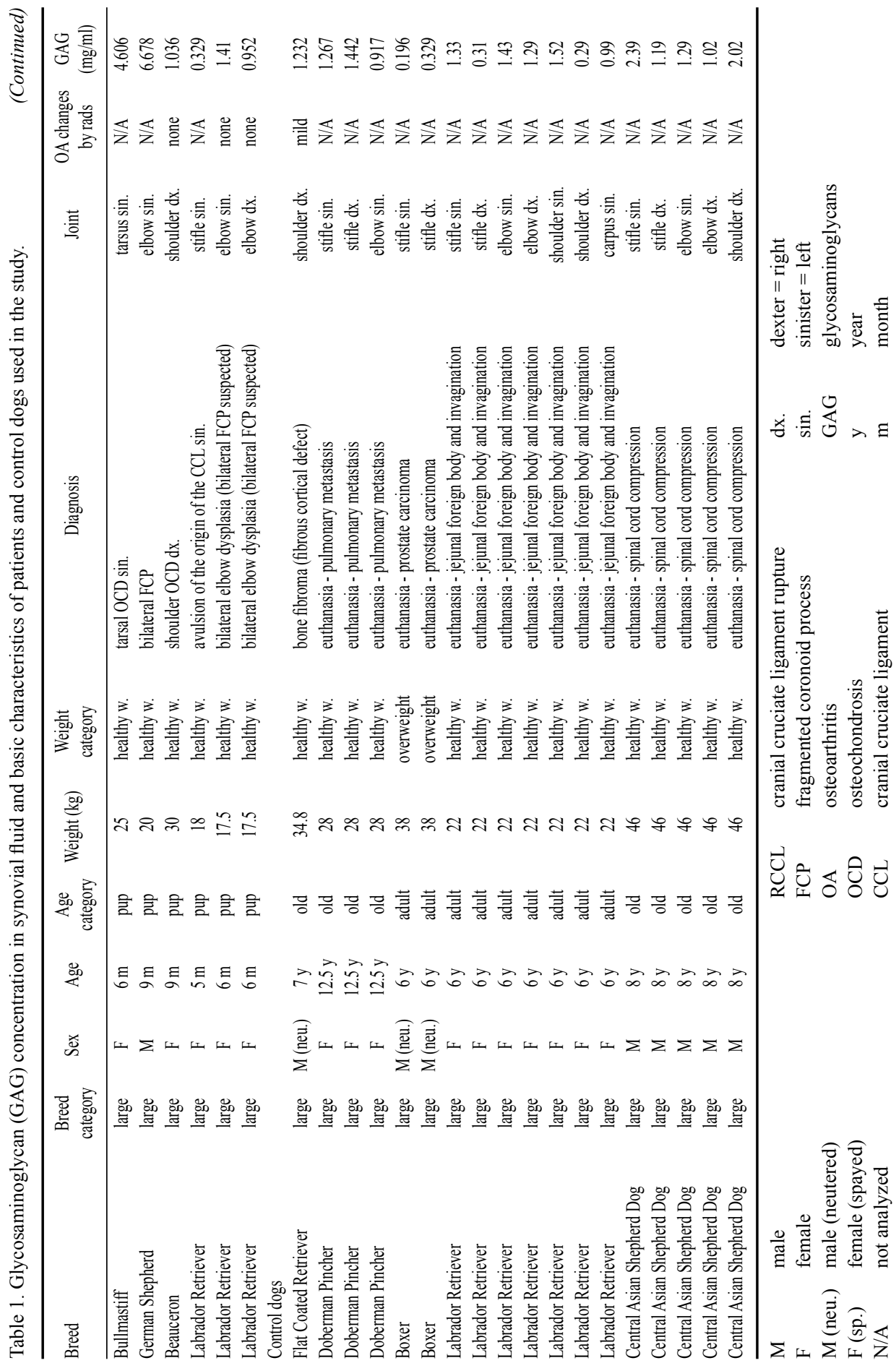




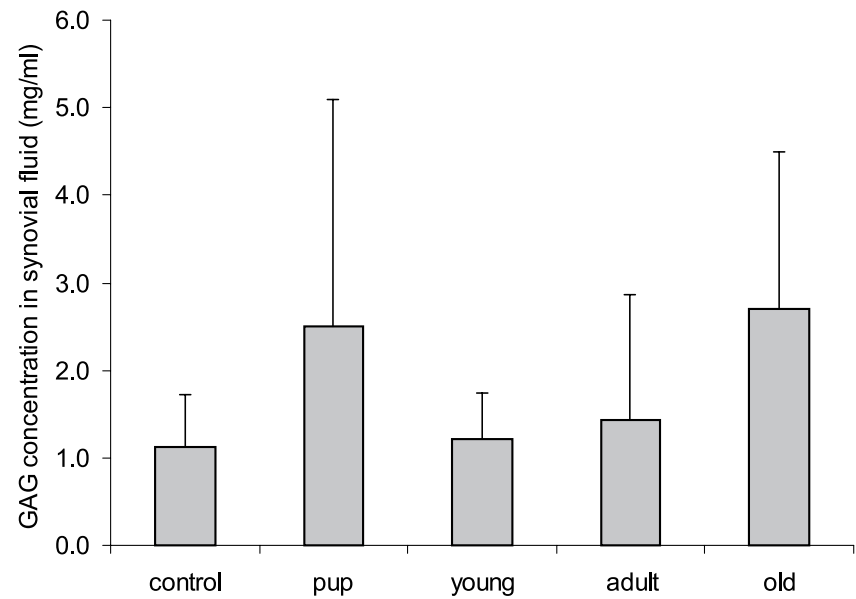

Fig. 2. Comparison of glycosaminoglycan (GAG) mean values among age groups of dogs. Control - dogs euthanized for disorders not affecting joints, $\mathrm{n}=18$ (joints); pup - dogs with joint disease ( 6 months and younger for small breeds, 9 months and younger for medium breeds, and 12 months and younger for large breeds), $\mathrm{n}=7$ (joints); young - dogs with joint disease with the ages of 6 - 12 months for small breeds, 9-18 months for medium breeds, and 12-30 months for large breeds, $\mathrm{n}=9$ (joints); adult - dogs with joint disease between the ages of 1-9 years for small breeds, 1.5-8 years for medium breeds, and 2.5-6 years for large breeds, $n=14$ (joints); old - dogs with joint disease with the age of 9 years and older for small breeds, 8 years and older for medium breeds, and 6 years and older for large breeds), $\mathrm{n}=5$ (joints).

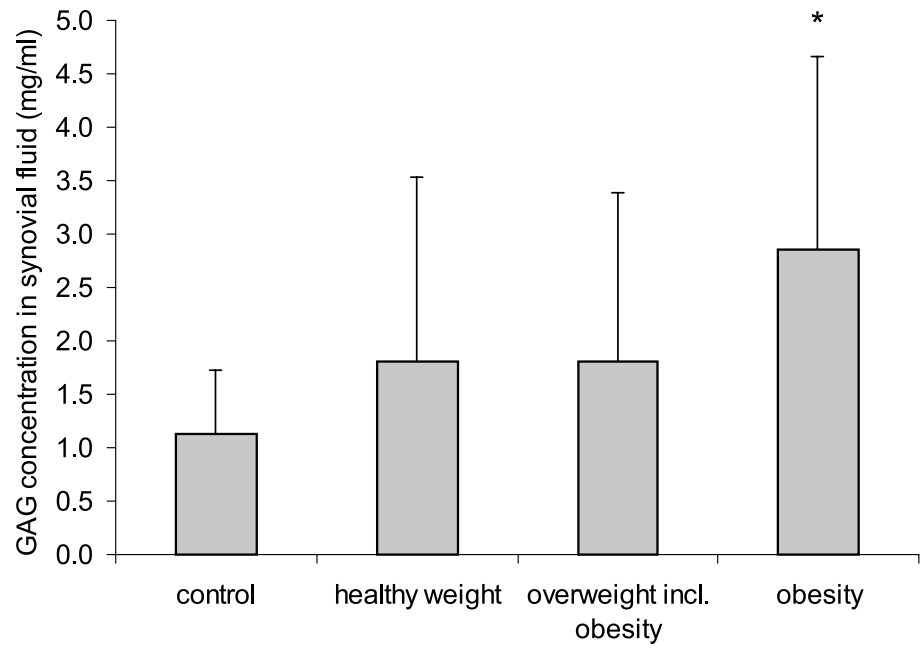

Fig. 3. Comparison of glycosaminoglycan (GAG) mean values among weight groups. Control - dogs euthanized for disorders not affecting joints with healthy weight, $\mathrm{n}=18$ (joints); healthy weight - dogs with joint disease meeting the breed standards of the Fédération Cynologique Internationale (FCI), $\mathrm{n}=26$ (joints); overweight dogs with joint disease exceeding FCI breed standards by $10-19 \%, \mathrm{n}=4$ (joints); obesity - dogs exceeding with joint disease FCI breed standards by $20 \%$ and more, $\mathrm{n}=5$ (joints). * significant difference $(P<0.05)$.

overweight dogs and changed social status of dogs in general have a gross impact on increasing the incidence of secondary OA among world canine population including the Czech Republic. 
The $\mathrm{OA}$ is a chronic degenerative joint disorder involving joint cartilage, underlying bone structures and the synovial membrane that interact together during degradation and reparation process (Owens and Biery 1999). The OA is considered a non-inflammatory arthropathy; its typical features are fragmentation and loss of joint cartilage, narrowing or even collapsing of joint space well seen on radiographs, increased subchondral density (sclerosis) and newly formed bone structures at the edge of joint surfaces (osteophytes) (Pedersen and Poole 1978). In this study, we focused on cartilage degradation products in SF, which are believed to be elevated during certain stages of OA.

In spite of the relatively low number of evaluated joints along with high data variability, some interesting conclusions could be drawn. The GAG concentrations in SF samples seem to be higher in dogs suffering from OA than in control dogs, regardless of their actual body weight. However, obese dogs reached a significant difference compared to control dogs. Although the finding supports the general idea of a relationship between the body weight and OA development in joints, there are no studies available specifically discussing the body weight versus GAG concentration in SF.

Also, there is a certain age influence seen as the old dogs and pups have the highest mean values and on the other hand, the lowest mean value was measured in young dogs group. A similar conclusion was made by authors evaluating the correlation of GAG concentration in SF and age in horses; the highest values were measured in a group of newborn foals and those values kept decreasing over the lifetime (van den Boom et al. 2004). Later, the same authors cast doubts on GAG measurement in early stages of cartilage damage when no obvious changes could be seen since those values were lower compared to values of horses with at least minimal visible OA changes (van den Boom et al. 2005). Negative correlations between GAG values in SF and cartilage damage stage were reported by other authors in horses, too (Fuller et al. 2001). The GAG concentration and severity of radiographic changes correlated negatively even in dogs (Innes et al. 1998) and there was no correlation between radiographic changes and GAG in human patients (Belcher et al. 1997). These indicators (severity of radiographic changes and GAG content in SF) did not correlate in our study either (data not shown). The elevated GAG and keratan sulphate concentrations were observed in the patients' SF samples during the acute process compared to the chronic disease. The authors explained this as a result of higher metabolic rate and final GAG depletion in joint cartilage content (Ratcliffe et al. 1988). The same fact was also confirmed in dogs throughout the early and late OA stages (Innes et al. 2005).

It may be considered that high GAG values are caused with a high metabolic rate in pups when anabolic processes significantly outnumber catabolic processes during the intensive phase of their growth; on the other hand, in the old dogs, catabolic processes that degrade cartilage tissue may also increase GAG in SF. Another explanation for this phenomenon can be an increased glycosylation of cartilage proteins in older animals, which leads to higher GAG concentration in SF (DeGroot et al. 2001).

The variability in age of dogs included in our study might explain the differences among groups based on primary joint disorder due to the fact that the FCP group, which achieved the absolutely highest mean value of GAG concentration in the whole study, was dominated by pups and old dogs (66\% of dogs diagnosed with FCP) but the RCCL group with the lowest mean GAG value was mainly formed from adult and young dogs $(81 \%)$ who had low GAG values. Nevertheless, the high FCP values in FCP group can be influenced by the fact that all samples were obtained from one compartment, the elbow joint exclusively. The GAG concentration may significantly differ among joints of a healthy animal (Fuller et al. 1996).

Finally, our study supports the widely-accepted dogma that obesity is a negative factor contributing to joint degradation process, manifested by an increased level of GAG released 
into samples of canine SF. Further evaluation is needed in order to answer the question how soon the obesity-dependent degradation starts and whether the process can be significantly slowed down after the individual has lost the abundant body weight.

\section{Acknowledgments}

This work was supported by the University of Veterinary and Pharmaceutical Sciences Brno, Czech Republic (Project IGA IG161663) and the Ministry of Agriculture of the Czech Republic (Project MZE 0002716202) with the grant of Internal Grant Agency of VFU Brno No IG161663 and the project of Ministry of Agriculture No MZE 0002716202

The authors thank Dr. Alicia Grasso for the kind manuscript proofreading.

\section{References}

Arican M, Carter SD, Bennett D, May C 1994: Measurement of glycosaminoglycans and keratan sulphate in canine arthropathies. Res Vet Sci 56: 290-297

Belcher C, Yaqub R, Fawthrop F, Bayliss M, Doherty M 1997: Synovial fluid chondroitin and keratan sulphate epitopes, glycosaminoglycans, and hyaluronan in arthritic and normal knees. Ann Rheum Dis 56: 299-307

Degroot J, Verzijl N, Wenting-Van Wijk MJ, Bank RA, Lafeber FP, Bijlsma JW, Tekoppele JM 2001: Age-related decrease in susceptibility of human articular cartilage to matrix metalloproteinase-mediated degradation: the role of advanced glycation end products. Arthritis Rheum 44: 2562-2571

Fuller CJ, Barr AR, Dieppe PA, Sharif M 1996: Variation of an epitope of keratan sulphate and total glycosaminoglycans in normal equine joints. Equine Vet J 28: 490-493

Fuller CJ, Barr AR, Sharif M, Dieppe PA 2001: Cross-sectional comparison of synovial fluid biochemical markers in equine osteoarthritis and the correlation of these markers with articular cartilage damage. Osteoarthr Cartil 9: $49-55$

Innes JF, Little CB, Hughes CE, Caterson B 2005: Products resulting from cleavage of the interglobular domain of aggrecan in samples of synovial fluid collected from dogs with early- and late-stage osteoarthritis. Am J Vet Res 66: 1679-1685

Innes JF, Sharif M, Barr AR 1998: Relations between biochemical markers of osteoarthritis and other disease parameters in a population of dogs with naturally acquired osteoarthritis of the genual joint. Am J Vet Res 59: $1530-1536$

Johnston SA 1997: Osteoarthritis. Vet Clin North Am Small Anim Pract 27: 699-720

Kempson GE, Muir H, Swanson WVA, Freeman MAR 1970: Correlations between stiffness and the chemical constituents of cartilage on the human femoral head. Biochem Biophys Acta 215: 70-77

Linsenmayer TF, Toole BP 1977: Biosynthesis of different collagens and glycosaminoglycans during limb development. Review. Birth Defects Orig Artic Ser 13: 19-35

Maroudas A, Bayliss MT, Venn MF 1980: Further studies on the composition of human femoral head cartilage. Ann Rheum Dis 39: $514-523$

Myers SL, O'Connor BL, Brandt KD 1996: Accelerated clearance of albumin from the osteoarthritic knee: implications for interpretation of concentrations of "cartilage markers" in synovial fluid. J Rheumatol 23: $1744-1748$

Owens JM, Biery DN 1999: Joints. In: Owens JM, Biery DN (Ed.): Radiographic interpretation for the small animal clinician. Williams and Wilkins, Baltimore, pp 59-103

Pedersen NC, Pool R 1978: Canine joint disease. Vet Clin North Am 8: 465-493

Ratcliffe A, Doherty M, Maini RN, Hardingham TE 1988: Increased concentrations of proteoglycan components in the synovial fluids of patients with acute but not chronic joint disease. Ann Rheum Dis 47: 826-832

Renberg WC 2005: Pathophysiology and management of arthritis. Vet Clin North Am Small Anim Pract 35: 1073-1091

Uebelhart D 2008: Clinical review of chondroitin sulfate in osteoarthritis. Osteoarthr Cartil 16 (Suppl 3): S19-S21

Van Den Boom R, Brama PA, Kiers GH, De Groot J, Van Weeren PR 2004: Assessment of the effects of age and joint disease on hydroxyproline and glycosaminoglycan concentrations in synovial fluid from the metacarpophalangeal joint of horses. Am J Vet Res 65: 296-302

Van Den Boom R, Van Der Harst MR, Brommer H, Brama PA, Barneveld A, Van Weeren PR, De Groot J 2005: Relationship between synovial fluid levels of glycosaminoglycans, hydroxyproline and general MMP activity and the presence and severity of articular cartilage change on the proximal articular surface of P1. Equine Vet J 37: 19-25 